\title{
Microvibrissae-Based Texture Discrimination
}

\author{
Praveen Kuruppath, ${ }^{\star}$ Erez Gugig, ${ }^{\star}$ and Rony Azouz \\ Department of Physiology and Cell Biology, Zlotowski Center for Neuroscience, Ben-Gurion University of the Negev, Beer-Sheva 84105, Israel
}

Rodents use their whiskers to detect a variety of tactile features of their environment. They do so by using two functionally distinct whisker systems: the macrovibrissae and microvibrissae. To determine the functional role of unexplored microvibrissae, we recorded from the cortical area representing the frontobuccal pad in anesthetized rats while presenting moving textures of varying coarseness. We find that surface coarseness is coded by the discharge rates of frontobuccal pad cortical neurons. Cortical neurons can use this response measure to robustly and reliably discriminate between the different textures. While neuronal discharge rates carry tactile information, the highly reproducible firing patterns of these neurons suggest that a single spike train may contain sufficient information to encode the stimulus. Together, these results indicate that rodents may use their microvibrissae to distinguish between surfaces having subtly different textures and shapes.

Key words: frontobuccal pad; somatosensory; textures; whisker

\section{Introduction}

Rodents use their whiskers to detect and distinguish a variety of tactile features in their environment (Kleinfeld et al., 2006), including object position (Mehta et al., 2007; Knutsen and Ahissar, 2009), shape (Brecht et al., 1997; Harvey et al., 2001), aperture and gap width (Krupa et al., 2001), and textures (Carvell and Simons, 1990; Diamond et al., 2008; Lottem and Azouz, 2009; Diamond, 2010; Jadhav and Feldman, 2010; Morita et al., 2011). Rodent whiskers are able to gather such a rich variety of tactile information by way of two functionally distinct whisker systems: the long, moveable macrovibrissae, which are swept across objects and surfaces (Sachdev et al., 2001; Berg and Kleinfeld, 2003), and shorter, nonmoveable microvibrissae. Most relevant studies have focused on the macrovibrissae and have shown that rodents can discriminate between different textures at high resolutions (Morita et al., 2011). However, this acuity cannot be explained by differences in firing rates in cortical barrel neurons, since it has been shown that they may account for detection of only large texture differences (Arabzadeh et al., 2005; Jadhav et al., 2009). Meanwhile, the functional role of the microvibrissae is only partially understood, since it has been shown that they are capable of mediating object recognition (Brecht et al., 1997), and since most behavioral studies of texture discrimination involved contact with both vibrissae (Carvell and Simons, 1990, 1995).

The present study explores whether the somatosensory cortex can code for surface coarseness when rodents sense the world through the microvibrissae. Our findings suggest that rodents

Received Oct. 2, 2013; revised Feb. 20, 2014; accepted Feb. $28,2014$.

Author contributions: R.A. designed research; P.K. performed research; E.G. analyzed data; R.A. wrote the paper. This work was supported by a grant from the Israel Science Foundation to R.A.

*P.K. and E.G. contributed equally to this work.

The authors declare no competing financial interests.

Correspondence should be addressed to Rony Azouz, PhD, Dept. of Physiology and Cell Biology, Faculty of Health

Sciences, Ben-Gurion University of the Negev, Beer-Sheva 84105, Israel. E-mail: razouz@bgu.ac.il.

DOI:10.1523/JNEUROSCI.4217-13.2014

Copyright $\odot 2014$ the authors $\quad 0270-6474 / 14 / 335115-06 \$ 15.00 / 0$ may use their frontobuccal pad (FBP) to discriminate between objects having different surface coarseness.

\section{Materials and Methods}

Whisker stimulation. Head angular velocities associated with rat exploration were taken from Lottem and Azouz (2009). We replayed these pad movements across different surfaces by covering a face of a rotating cylinder with sandpaper of different grades of coarseness and rotating the wheel against the pad (Fig. $1 B ; n=11$ animals; $n=19$ neurons). The wheel face was placed so that the microvibrissae rested upon it (Fig. 1B). The wheel was placed to mimic rotational head movements. The angular velocity was controlled using a DC motor driven at $\sim 39 \mathrm{~mm} / \mathrm{s}$ to replicate median head velocity. The 30-mm-diameter wheel was driven by a DC motor (Farnell). We used surfaces of eight different grades from coarse grained to fine grained (the numbers in the parentheses indicate the average grain diameter): P100 $(162 \mu \mathrm{m}), \mathrm{P} 320(46 \mu \mathrm{m}), \mathrm{P} 400(35$ $\mu \mathrm{m})$, P500 $(30 \mu \mathrm{m})$, P600 $(26 \mu \mathrm{m})$, P800 $(22 \mu \mathrm{m})$, P1000 $(18 \mu \mathrm{m})$, P1200 $(15 \mu \mathrm{m})$. These grades were chosen in accordance with previous studies (Arabzadeh et al., 2005; Hipp et al., 2006; Morita et al., 2011). For each texture, we recorded 50 trials per texture of the rotating cylinder, each lasting $\sim 1 \mathrm{~s}$.

To measure surface coarseness, we used a calibrated noncontact optical displacement measuring system (resolution, $1 \mu \mathrm{m}$; sampled region, $200 \mu \mathrm{m}$; LD1605-2, Micro-Epsilon). We rotated the texture-covered cylinders at velocities corresponding to head movements (see above) and measured the height of surface microfeatures at one point on the texture over time as the surface rotates (Fig. $1 G$, bottom). We then replayed these diverse distance profiles through a galvanometer stimulator to the FBP (Fig. $1 C ; n=8$ animals; $n=52$ neurons). A plate $(5 \times 5 \mathrm{~mm})$ was attached to the end of a rod, which was attached to the axis of the galvanometer. The plate was lying close to the pad.

Surgical procedures and recording. Adult male Sprague Dawley rats ( $n=19 ; 250-350 \mathrm{~g})$ were used. Animal surgeries and in vivo recordings were performed as previously described (Lottem and Azouz, 2009). All experiments were conducted in accordance with international and institutional standards for the care and use of animals in research.

After placing subjects in a stereotactic apparatus (TSE Systems), an opening was made in the skull overlying the FBP cortex (mediolateral, $6.2-7.2 \mathrm{~mm}$; anteroposterior, $0.8-2.5 \mathrm{~mm}$ ), and tungsten microelectrodes ( $2 \mathrm{M} \Omega$; We Sense) were lowered until units drivable by pad stim- 
ulations were encountered. The recorded signals were amplified $(1000 \times)$, bandpass filtered $(0.1-10 \mathrm{kHz})$, digitized $(25 \mathrm{kHz})$, and stored for off-line spike sorting and analysis. Spike extraction and sorting was accomplished with MClust (by A.D. Redish; available from http://redishlab.neuroscience.umn.edu/MClust/ MClust.html), which is spike-sorting software based on Matlab (Mathworks). The extracted and sorted spikes were stored at a $0.2 \mathrm{~ms}$ resolution and peristimulus time histograms (PSTHs) were computed.

Data analysis. The coarseness of each surface was characterized by its power spectrum (Lottem and Azouz, 2009). Briefly, for each replayed texture signal we estimated the power spectrum by the modulus squared of the Fourier transform.

We used signal detection theory [receiver operating characteristics, receiver-operator curve (ROC) analysis; Green and Swets, 1974] to compute the probability that an ideal observer could accurately report the difference between different textures based on neuronal activity. For each texture pair, the ROC was constructed. To transform the raw data into a measure of discriminability, we analyzed the distributions of discharge rates across trials. The area under the ROC (AUC) corresponds to the performance expected of an ideal observer in a two-alternative, forced-choice paradigm, as the one used in the present analysis. It approaches 0.5 for a chance level discrimination, and equals 1 for a perfect discrimination.

Spike-triggered averaging (STA) of texture distance profiles were calculated from DCsubtracted texture signals. We calculated STA signal for each texture for each neuron separately. The STAs were calculated between \pm 50 ms. Significance of an event preceding a spike was determined by crossing a threshold (mean $+3 \mathrm{SD}$ ) and lies $<15 \mathrm{~ms}$ before the spike.

The reliability measure (RM) is calculated only on neurons that were stimulated with random repeated parts of the textures (frozen, $n=$ 10). The RM is based on Mainen and Sejenowski (1995). Briefly, we detected in each PSTH (bin size, $5 \mathrm{~ms}$ ), peaks that exceed a certain threshold. The threshold was calculated for each neuron and texture separately. Threshold calculation is based on the mean \pm SD of peak values of the PSTH. We then calculated the cumulative sum of all the probability values within events that crossed the threshold, divided by the cumulative sum of all the bins in total. To calculate the significance level of RM, we created for each neuron a surrogate dataset in which we shuffled the interspike interval. We defined a neuron as reliable if, in response to at least one of its textures, RM was above a significant level (8 of 10).

To determine the how well each neuron can decode the presented stimulus, we created a prediction of the stimulus from the recorded responses. We computed an STA for stimulus-response pairs recorded during the first half of each texture stimulus. We then convolved this STA with each spike train recorded during the second half of the stimulus to derive the prediction. We estimated the accuracy of this prediction by computing the Pearson's correlation coefficient between the actual and the predicted stimuli. The results are presented as the mean \pm SEM.

\section{Results}

Here, we test the ability of microvibrissae to mediate texture discrimination, and quantitatively evaluate the neuronal mechanisms underlying this discrimination (Fig. 1A). To examine how
G

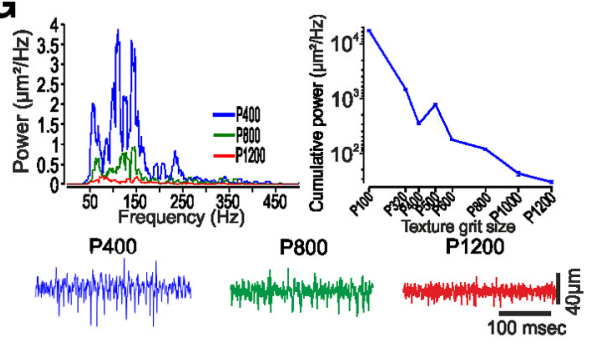

H

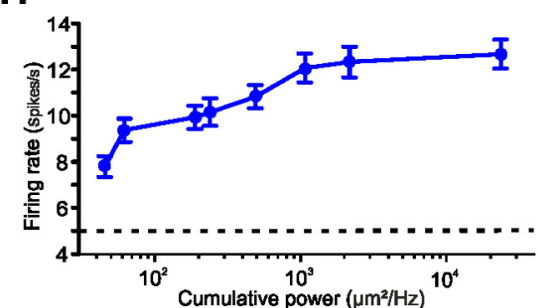

I

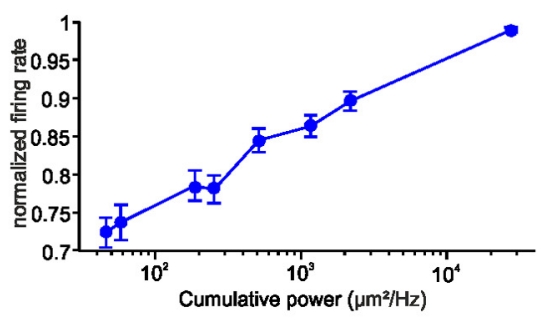

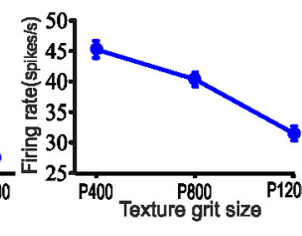

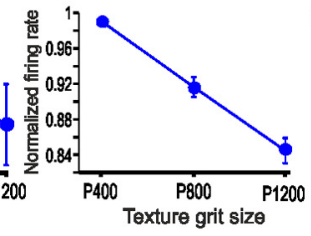

(1)

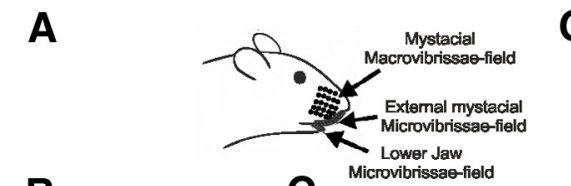

Figure 1. The influence of surface coarseness on neuronal firing rates. $A$, Side view of the mystacial whisker fields. $B, C$, and a galvanometer (C.D, PSTHs of two cortical neurons to the different textures and influence of surface

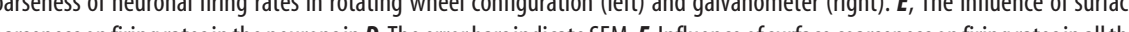
eurons in the two stimulus paradigms. Error bars are SEM. G, Smoothed power spectra (moving mean) of distance profiles across textures of the textures shown in bottom. The right panel shows the relation between the cumulative power $(\mathrm{CP} ; 50-500 \mathrm{~Hz})$ and surface coarseness. $\boldsymbol{H}$, The influence of $\mathbf{C P}$ on firing rates in the neuron in $\boldsymbol{D}$ (left). The dashed horizontal line indicated spontaneous activity. $I$, The influence of $C P$ on normalized firing rates in all neurons.

surface coarseness is transformed into neuronal discharges, we took two approaches in which we mimicked the interaction between different surfaces and the FBP while the animal is moving freely (see Materials and Methods; Fig. $1 B, C$ ). In the first approach, we quantified pad velocity associated with head movements (Lottem and Azouz, 2009). We then replayed these pad movements across different surfaces by covering a face of a rotating cylinder with sandpaper of different grades of coarseness and rotating the wheel against the pad (see Materials and Methods; Fig. $1 B$ ). In the second approach, we scanned each of the surfaces and transformed texture surface into a unique distance profile (see Materials and Methods; Fig. 1G, bottom). We then replayed these diverse distance profiles using a galvanometer stimulator to the FBP (Fig. 1C). Cortical responses to these two types of stimuli are shown in Figure $1 B, C$. These results indicate that surface coarseness is translated into neuronal discharge rates. Surface coarseness reduction led to a reduction in discharge rates (Fig. $1 E, F)$.

Instead of relying on an industrial measure of grit size, we measured the actual surface coarseness of each surface. We did it by measuring the distance profile of each surface and then calcu- 


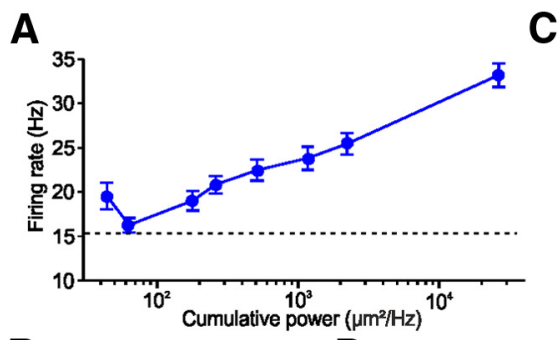

B

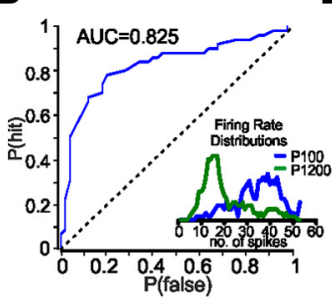

D

$\mathbf{F}$

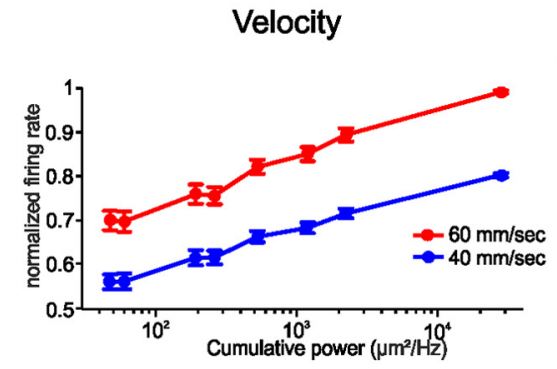

G

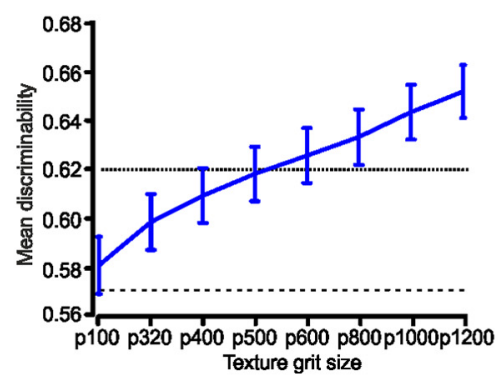

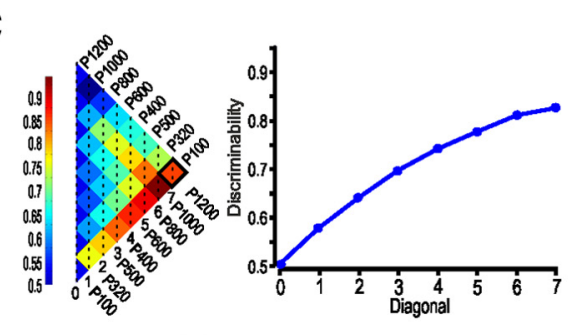

$E$

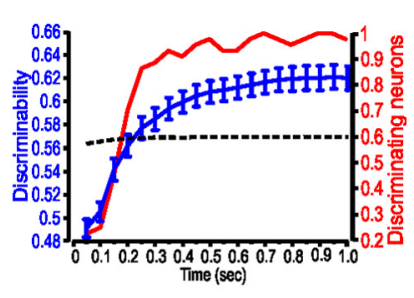

Direction

H

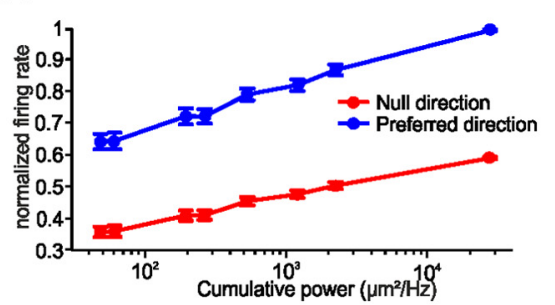

I

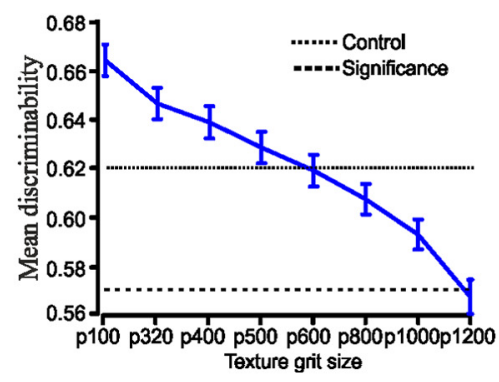

Figure 2. Texture discrimination using ROC analysis. $\boldsymbol{A}$, An example of the influence of cumulative power on firing rates in a single cortical neuron. $\boldsymbol{B}, \mathrm{ROC}$ used to discriminate between the two textures (P100 vs P1200). The inset shows the distribution of neuronal firing rates of the neuron in $A$ to two textures. $C$, The area under the ROC values, using firing rates, for all pairs of textures. Averaging all values along the diagonal results in a discriminability plot for the neuron in $A$. D, Discriminability plot for all neurons in the study. The dashed horizontal line indicates the significance level (see corresponding text). $\boldsymbol{E}$, The influence of stimulus duration on texture discriminability and the number of significant neurons. The dashed line indicates the significance level. $\boldsymbol{F}, \boldsymbol{H}$, The influence of head velocity and direction on texture discriminability. Normalized changes in firing rates as a function of head velocity and head direction. $G, I$, The influence of changing the velocity and head direction on each of the textures, on the average discriminability. The dotted line is the control conditions. The dashed line is significance level. The error bars indicate SEM.

lating the fast Fourier transform of these profiles. We quantitatively defined surface coarseness by computing the cumulative power of the distance profile power spectrum of all surfaces (Fig. $1 E)$. The coarser the surface is, the higher the power. Our results show that reducing surface coarseness resulted in an overall reduction of the $50-500 \mathrm{~Hz}$ power, which will result in decreased cortical neuronal firing rates and vice versa (Fig. $1 \mathrm{H}, I$ ).

To determine whether neuronal firing rates can encode for surface coarseness, we used the ideal observer approach. We first calculated the distribution of firing rates as a function of surface coarseness (Fig. 2B, inset). We then used the ROC analysis (Green and Swets, 1974) to assess the discriminative power of surface coarseness-dependent firing rates. The ROC in Figure $2 B$, which is used to discriminate between two stimuli (P100 and P1200), shows that neuronal discharge rates are discriminating between the two textures. To assess the discrimination between all textures pairs, we calculated the AUC for all textures pairs. This is shown in the left panel of Figure $2 C$. Each pixel in this matrix represents an AUC value for a pair of textures. To reduce the dimensionality of our analysis, we average all matrix values that belong to the same diagonal (average global AUC, or discriminability plot; Figure $2 C$, right), which illustrates the overall discrimination for all texture differences. Significance levels were assessed by intershuffling trials randomly from different textures to build the surrogate responses, which lack the texture-response correlations, and calculate their AUC values. We repeated this process for 1000 iterations, from which we extract the value for the $90 \%$. This value serves as the significance level. We consider a neuron as possibly discriminating one if at least one of points in the discriminability plot values was above significant level. Of 52 units, 44 $(\sim 85 \%)$ were found to be possibly discriminating. Figure $2 D$ shows the mean discriminability plot of these neurons.

As an extension of these results, we sought to determine the temporal constraints on texture discrimination. We examined the influence of response duration on texture discrimination by changing the relevant time window in which we measure the firing rates. To assess the time scale needed for the neurons to discriminate between different textures, we calculated for each neuron the discriminability based on the response occurring on different time windows from stimulus onset. We measured the discriminability between different time scales with jumps of $50 \mathrm{~ms}$. The time-scale analysis was made only on neurons we consider as possibly discriminating neurons $(n=44, \sim 85 \%$ of the total population). Significance values were calculated separately for each timewindow discriminability measure. We found that texture discrimination becomes significant after $\sim 200 \mathrm{~ms}$. At this point in time, the majority of the recorded neurons becomes significant (Fig. 2E). These results suggest that this neuronal constraint may enable the animal to discriminate between textures using a single pass of the FBP.

Finally, one of the major limitations in sensing the environment is stimulus generalization. Specifically, what happens to texture discrimination if the animal encounters several of the textures at a different head velocity or direction? These types of mutability may cause a change in neuronal discharge rates, which may have critical detrimental effect on the quality of the discrimination. We tested for the influence changes in pad velocity and direction on neuronal discharge rate in cortical neurons by varying the velocity of the textured-covered wheel from $\approx 40$ to $\approx 60$ 
A

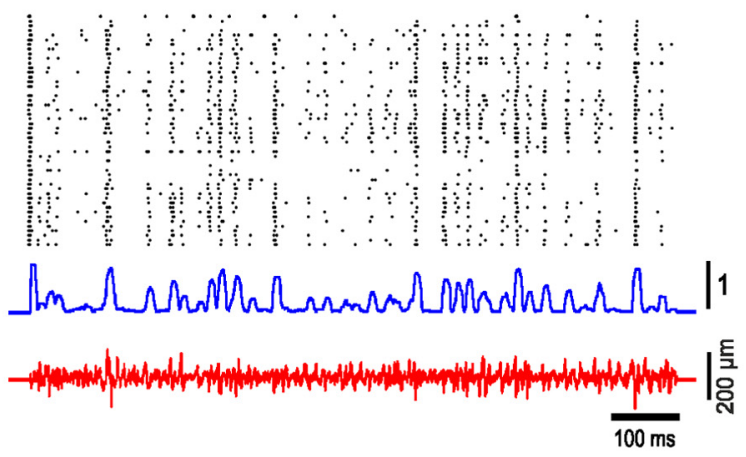

B
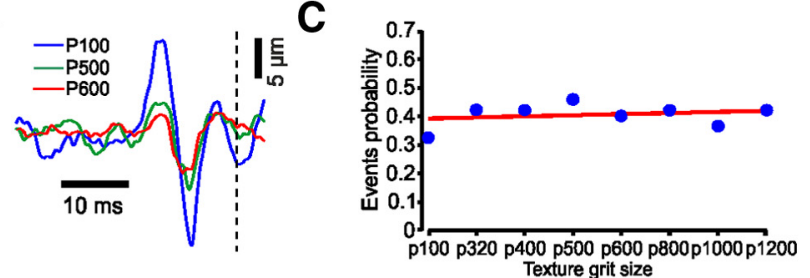

E

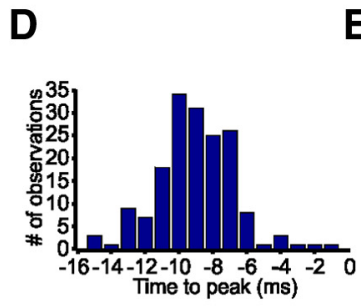

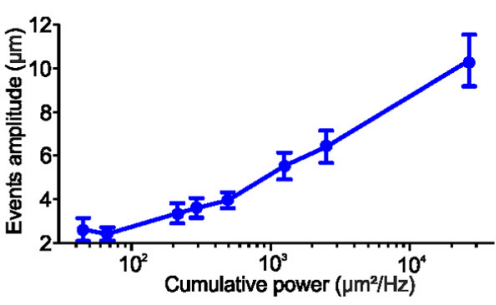

$\mathbf{F}$

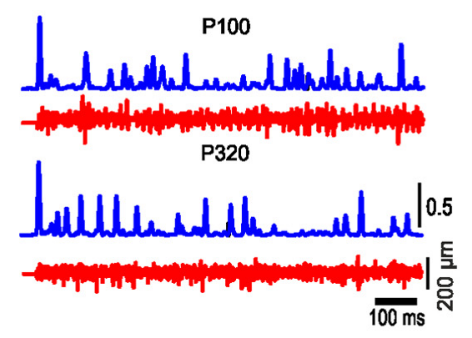

G
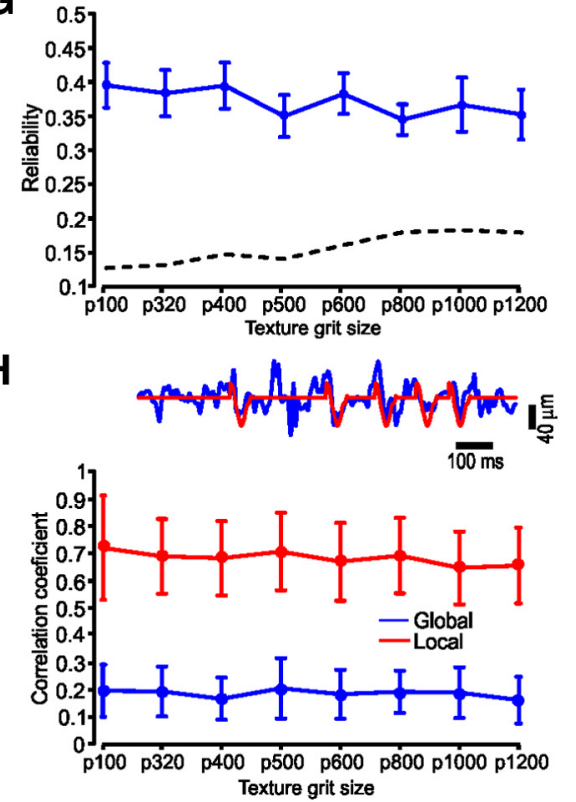

Figure 3. Cortical neurons respond to texture stimuli with highly reproducible spike train. $\boldsymbol{A}$, Raster (top), PSTH (middle), and the frozen segment texture stimulus, P100 (bottom). $\boldsymbol{B}$, STA of different textures. C, Proportion of neurons showing significant STA is not dependent on textures. D, Distribution of times between spike generation and peak of the STA. $\boldsymbol{E}$, The influence of cumulative power on STA amplitude. $\boldsymbol{F}$, Different temporal response pattern to different textures. $\boldsymbol{G}$, Response reliability is not dependent on texture. The dashed line indicates the significance threshold. $\boldsymbol{H}$, Global and local correlation coefficients are not dependent on texture. The upper trace shows a segment of the actual (blue) and predicted response (red). The error bars indicate SEM.

$\mathrm{mm} / \mathrm{s}$, and moved the wheel in the opposite direction. To test the influence of changes in pad velocity and direction, we compared the firing rates for three textures (p350, p600, and p1200) in the four conditions $(n=17)$. For each texture, we determined for each neuron whether changes in velocity or direction resulted in significant changes in firing rates (two-sample $t$ test, $\alpha=0.05$, one tailed). We found that an increase in pad velocity enhanced firing rates significantly in 54\% (p350), 65\% (p600), and 83\% (p1200) in the neurons in the different textures, while a change in direction resulted in a significant change in firing rates in $53 \%$ of the neurons. For the neurons that show significant increase in firing rates, rates scaled up by a factor of $1.24 \pm 0.46$ (Fig. $2 F$ ), while switching to null direction resulted in a decrease by a factor of $0.65 \pm 0.23$ in firing rates (Fig. $2 H$ ).

We used these factors to scale the responses to the galvanometer stimulator. For each trial in response to each texture of each neuron (only discriminating neurons), we scaled the firing rates according to changes in pad velocity and direction for each texture. We then calculated the average value of all points on the discriminability plot for control condition and for plots in which the firing rates of each texture were scaled separately according to the change in firing rates as a result of velocity or direction change. The average value in relation to the control is shown in Figure 2G,I. The results show that stimulus variation has both detrimental and favorable effects on discrimination, depending on the specific texture. Altogether, texture discrimination remains mostly significant. Our results indicate that, within the stimulus variation we have tested, cortical neuron resilience to stimulus noise is important in relaying texture signals, thus making the use of the FBP plausible for texture discrimination.

There is mounting evidence that sensory perception involves temporal coding schemes in which the timing of individual spikes encodes information (Zador, 1997; Dayan and Abbott, 2001). To test for the plausibility of temporal encoding in our system, we chose a random sequence of textures and repeated it multiple times (Fig. 3A). Using STA of surface distance for all spikes in a session reveals that action potentials in cortical neurons commonly arise from high-frequency changes in surface structure (Fig. 3B). According to a significance criteria (see Materials and Methods), 43 of the 52 ( $83 \%$ ) neurons have at least one texture that yields significant STA. In these neurons, $49 \%$ of the textures on average have significant STA. We calculated the probability of an STA event to occur in a specific texture. We found no correlation between the texture and the probability of having an STA event. Linear regression between the probabilities of the different textures was flat $\left(r^{2}=0.05\right.$; slope, 0.004; Fig. $\left.3 C\right)$. These events occur 8-10 ms before spike generation and their amplitude is highly dependent on surface coarseness (Fig. $3 D, E$ ), while the proportions of neurons responding to these events are not dependent on surface coarseness (Fig. $3 C$ ). These highly reproducible firing patterns suggest that a single spike train may contain sufficient information to encode the stimulus. We first calculated response reliability (Mainen and Sejnowski, 1995) and found that 
while reliability measure does not depend on texture coarseness, a large proportion of the spikes respond reliably to the same feature (Fig. 3G; see Materials and Methods). We then attempted to predict the stimulus from the recorded responses. We computed the STA for stimulus-response pairs recorded during the first half of the stimulus. We then convolved this STA with each spike train recorded during the second half of the stimulus to derive the prediction. We estimated the accuracy of this prediction by computing the Pearson's correlation coefficient between the actual and the predicted stimuli. This process is illustrated for one neuron in Figure $3 H$ (top). Since the neuronal firing rates are relatively low, the original stimulus features (blue trace) are only partially captured by the predicted stimulus (red). We therefore created two measures of prediction: global and local. The global prediction is the Pearson's correlation coefficient between the complete actual and the predicted stimuli (mean, 0.36), while the local prediction is the Pearson's correlation coefficient only during spike occurrences (mean, 0.816). Together, our results show for the first time that FBP neurons may be used to distinguish between surfaces having subtly different textures and shapes.

\section{Discussion}

Our results show that rats can sense surface texture using their FBP. They may do so by brushing their FBP against different surfaces for relatively short durations, which may correspond to touch duration with the macrovibrissae (von Heimendahl et al., 2007). These sweepings of the pad against different textures transform surface coarseness into neuronal discharge rates, which can be used to discriminate between different textures as well as sensation of object shape (Brecht et al., 1997). This and previous studies suggest that microvibrissae can provide salient texture information as good as the coarsely spaced macrovibrissae (Brecht et al., 1997; Diamond et al., 2008; Morita et al., 2011). Texture discrimination is robust and can endure changes in head velocity and direction. A third important finding in the study is the high temporal precision of responses to repeated presentations of arbitrary texture segments. This suggests that a single presentation of a stimulus provides sufficient information to encode complex stimulus features. Such a robust coding mechanism may provide a parallel coding scheme that may enable rodents to use their FBP to accurately discriminate between different textures (Arabzadeh et al., 2006).

Before discussing the implications of these findings, it is important to consider the set of assumptions upon which the interpretations are based. A critical caveat in the current study involves surface contact mimicry by using the galvanometer stimulator. This was done because of technical limitation. To validate the use of the two methods, in some of the neurons in the study we compared the responses to the two configurations and did not find any significant changes between the results. Finally, we are using anesthetized animals. This method has several disadvantages and advantages. While the magnitudes and correlations of cortical neuronal activity are significantly different from behaving animals, certain characteristics of cortical sensory processing, such as texture coding, are consistent across various brain states, including anesthesia (Arabzadeh et al., 2005). In contrast, the use of anesthetized animals offers the advantages of greater control on stimulus presentation, better single-unit separation, and more precision in placements for neuronal recordings.

\section{Texture discrimination through microvibrissae and macrovibrissae}

Except for two studies (Brecht et al., 1997; Morita et al., 2011), the literature on how rats detect a variety of tactile features of their environment concentrates mostly on the macrovibrissae (see Introduction). These two studies compared the functional significance of these two systems and concluded that macrovibrissae and microvibrissae both are capable of mediating high acuity form discrimination, while other studies have shown that macrovibrissae are also involved in detecting object position, shape, aperture, and gap width (see Introduction).

The current study has several major implications on the different theories of neural coding of texture coding (Diamond, 2010; Jadhav and Feldman, 2010). In one model, surface coarseness is related to the mean speed or total power of surfaceinduced whisker vibrations, which is encoded in mean firing rate of somatosensory cortex (S1) neurons (Arabzadeh et al., 2005; von Heimendahl et al., 2007). While we cannot measure microvibrissae velocity in response to the different textures, the increase in distance profile power as a function of texture coarseness suggests a monotonic dependence of neuronal firing rates on this power, an idea that contrasts with findings of previous macrovibrissae studies (Arabzadeh et al., 2005; von Heimendahl et al., 2007; Jadhav et al., 2009), which only show differences in firing rates between smooth and rough surfaces. This suggests that the mean speed model may account for detection of fine texture differences. In an extension of this theory, surface coarseness is encoded by the rate of high-velocity/acceleration "stick-slip" whisker micromotion events (Arabzadeh et al., 2005; Lottem and Azouz, 2008; Wolfe et al., 2008; Jadhav et al., 2009). Thus, surface coarseness is expressed in the rate of stick-slip events, which are encoded by the mean firing rate of $S 1$ neurons. Again, our results suggest that most of the neurons ( $83 \%$ ) in the FBP cortex respond precisely to high-velocity/acceleration events in $\sim 50 \%$ of the textures. Hence, the high-velocity/acceleration whisker micromotion model can also explain detection of relatively fine texture differences. Thus, the current study has implications for the way rodents sense their environment. By showing that the FBP cortex can code for surface coarseness, our results support the proposal put out by several studies that rodents may dynamically sense their environment by using both vibrissae systems to optimize their detection and discriminating strategies.

\section{References}

Arabzadeh E, Zorzin E, Diamond ME (2005) Neuronal encoding of texture in the whisker sensory pathway. PLoS Biol 3:e17. CrossRef Medline

Arabzadeh E, Panzeri S, Diamond ME (2006) Deciphering the spike train of a sensory neuron: counts and temporal patterns in the rat whisker pathway. J Neurosci 26:9216-9226. CrossRef Medline

Berg RW, Kleinfeld D (2003) Rhythmic whisking by rat: retraction as well as protraction of the vibrissae is under active muscular control. J Neurophysiol 89:104-117. Medline

Brecht M, Preilowski B, Merzenich MM (1997) Functional architecture of the mystacial vibrissae. Behav Brain Res 84:81-97. CrossRef Medline

Carvell GE, Simons DJ (1990) Biometric analyses of vibrissal tactile discrimination in the rat. J Neurosci 10:2638-2648. Medline

Carvell GE, Simons DJ (1995) Task- and subject-related differences in sensorimotor behavior during active touch. Somatosens Mot Res 12:1-9. CrossRef Medline

Dayan P, Abbott LF (2001) Theoretical neuroscience: computational and mathematical modeling of neural systems. Cambridge, MA: Massachusetts Institute of Technology.

Diamond ME (2010) Texture sensation through the fingertips and the whiskers. Curr Opin Neurobiol 20:319-327. CrossRef Medline

Diamond ME, von Heimendahl M, Knutsen PM, Kleinfeld D, Ahissar E 
(2008) 'Where' and 'what' in the whisker sensorimotor system. Nat Rev Neurosci 9:601-612. CrossRef Medline

Green DM, Swets JA (1974) Signal detection theory and psychophysics. Huntington, NY: R.E. Krieger.

Harvey MA, Bermejo R, Zeigler HP (2001) Discriminative whisking in the head-fixed rat: optoelectronic monitoring during tactile detection and discrimination tasks. Somatosen Mot Res 18:211-222. CrossRef Medline

Hipp J, Arabzadeh E, Zorzin E, Conradt J, Kayser C, Diamond ME, König P (2006) Texture signals in whisker vibrations. J Neurophysiol 95:17921799. CrossRef Medline

Jadhav SP, Feldman DE (2010) Texture coding in the whisker system. Curr Opin Neurobiol 20:313-318. CrossRef Medline

Jadhav SP, Wolfe J, Feldman DE (2009) Sparse temporal coding of elementary tactile features during active whisker sensation. Nat Neurosci 12:792800. CrossRef Medline

Kleinfeld D, Ahissar E, Diamond ME (2006) Active sensation: insights from the rodent vibrissa sensorimotor system. Curr Opin Neurobiol 16:435444. CrossRef Medline

Knutsen PM, Ahissar E (2009) Orthogonal coding of object location. Trends Neurosci 32:101-109. CrossRef Medline

Krupa DJ, Matell MS, Brisben AJ, Oliveira LM, Nicolelis MA (2001) Behavioral properties of the trigeminal somatosensory system in rats performing whisker-dependent tactile discriminations. J Neurosci 21:5752-5763. Medline
Lottem E, Azouz R (2008) Dynamic translation of surface coarseness into whisker vibrations. J Neurophysiol 100:2852-2865. CrossRef Medline

Lottem E, Azouz R (2009) Mechanisms of tactile information transmission through whisker vibrations. J Neurosci 29:11686-11697. CrossRef Medline

Mainen ZF, Sejnowski TJ (1995) Reliability of spike timing in neocortical neurons. Science 268:1503-1506. CrossRef Medline

Mehta SB, Whitmer D, Figueroa R, Williams BA, Kleinfeld D (2007) Active spatial perception in the vibrissa scanning sensorimotor system. PLoS Biol 5:e15. CrossRef Medline

Morita T, Kang H, Wolfe J, Jadhav SP, Feldman DE (2011) Psychometric curve and behavioral strategies for whisker-based texture discrimination in rats. PloS One 6:e20437. CrossRef Medline

Sachdev RN, Sellien H, Ebner F (2001) Temporal organization of multiwhisker contact in rats. Somatosen Mot Res 18:91-100. CrossRef Medline

von Heimendahl M, Itskov PM, Arabzadeh E, Diamond ME (2007) Neuronal activity in rat barrel cortex underlying texture discrimination. PLoS Biol 5:e305. CrossRef Medline

Wolfe J, Hill DN, Pahlavan S, Drew PJ, Kleinfeld D, Feldman DE (2008) Texture coding in the rat whisker system: slip-stick versus differential resonance. PLoS Biol 6:e215. CrossRef Medline

Zador A (1997) Spikes. Exploring the neural code—-Rieke, F, Warland, D, DeRuyter, R, Stevenick, V, Bialek, W. Science 277:772. 